\title{
The role of autophagy in the radiosensitivity of the radioresistant human nasopharyngeal carcinoma cell line CNE-2R
}

This article was published in the following Dove Press journal:

Cancer Management and Research

\author{
Zhong-Guo Liang* \\ Guo-Xiang Lin* \\ Bin-Bin Yu* \\ Fang Su \\ Ling Li \\ Song Qu \\ Xiao-Dong Zhu
}

Department of Radiation Oncology,

The Affiliated Tumor Hospital

of Guangxi Medical University,

Cancer Institute of Guangxi Zhuang

Autonomous Region, Nanning,

People's Republic of China

*These authors contributed equally to this work
Correspondence: Xiao-Dong Zhu

Department of Radiation Oncology, The Affiliated Tumor Hospital of Guangxi

Medical University, Cancer Institute of

Guangxi Zhuang Autonomous Region, 71

He Di Road, Nanning 53002I, People's

Republic of China

Tel +86 77l 533 I466

Email zhuxdonggxmu@।26.com
Purpose: The present study aimed to study the role of autophagy in the radiosensitivity of the radioresistant human nasopharyngeal carcinoma cell line CNE-2R.

Methods: Before being irradiated, CNE-2R cells were treated with the autophagy inhibitor chloroquine diphosphate (CDP) or the autophagy inducer rapamycin (RAPA). Microtubuleassociated protein light chain 3 (LC3-II) and p62 were assessed using Western blotting analysis 48 hours after CNE-2R cells were irradiated. The percentage of apoptotic cells was assessed via flow cytometry. CNE-2R cell viability was evaluated using the Cell Counting Kit-8 (CCK8). The radiosensitivity of cells was assessed via clone formation analysis.

Results: The level of autophagy in CNE-2R cells improved as the radiation dose increased, reaching the maximum at a dose of $10 \mathrm{~Gy}$. Autophagy was most significantly inhibited by 60 $\mu \mathrm{mol} / \mathrm{L}$ CDP in CNE-2R cells, but was obviously enhanced by $100 \mathrm{nmol} / \mathrm{L}$ RAPA. Compared with the irradiation (IR) alone group, in the IR + CDP group, autophagy was significantly inhibited, viability was low, the rate of radiation-induced apoptosis was increased, and radiosensitivity was upregulated. In contrast, cells of the IR + RAPA group exhibited greater autophagy, higher viability, a lower rate of radiation-induced apoptosis, and downregulated radiosensitivity.

Conclusion: The autophagy level is negatively correlated with radiosensitivity for the radioresistant human nasopharyngeal carcinoma cell line CNE-2R.

Keywords: nasopharyngeal carcinoma, autophagy, radiosensitivity

\section{Introduction}

Nasopharyngeal carcinoma (NPC) is endemic in southern China, southeast Asia, and northern Africa. ${ }^{1,2}$ According to a survey from the International Agency for Research on Cancer, there were an estimated 86,700 new cases and 50,800 NPC-related deaths in 2012. ${ }^{1}$ Radiotherapy (RT) is the primary treatment option. Due to advances in disease management, diagnostic imaging, radiotherapy technology, and the broader application of systemic therapy, the prognosis of NPC has improved significantly. 3,4 Nevertheless, local recurrence and distant metastasis after RT remain the main failure patterns in NPC and are closely associated with the radioresistance of NPC.,6 Therefore, an urgent need exists to identify an approach that can eliminate or reverse radioresistance in NPC cells.

In cancer cells, autophagy is a conserved response to various anticancer therapies, including chemotherapy and radiotherapy. ${ }^{7}$ Autophagy is characterized by the sequestration of bulk cytoplasm and organelles in double- or multimembrane autophagic vesicles as well as the delivery of these vesicles to, and subsequent degradation by, 
the lysosomal system of the cell. The process starts with the formation of the autophagosome or autophagic vacuole. Many studies have shown that autophagy is a basis for the radioresistance of various tumors, including lung cancer, ${ }^{8}$ hepatocellular carcinoma, ${ }^{9}$ and colorectal cancer. ${ }^{10}$

In our previous study, we found that autophagic inhibition could enhance the radiosensitivity of the NPC cell line CNE-2. ${ }^{11}$ In addition, we used fractionated radiation in vitro to construct a radioresistant NPC (CNE-2R) cell line. ${ }^{12}$ To our knowledge, few studies have demonstrated the relationship between autophagy and the radioresistance of CNE-2R. We hypothesized that the level of autophagy had a negative correlation with the radiosensitivity of CNE-2R. In this study, we have examined the role of autophagy in the radiosensitivity of the radioresistant human NPC cell line CNE-2R.

\section{Materials and methods Cell culture}

A human NPC cell line, CNE-2, which was constructed by the Cancer Hospital of Chinese Academy of Medical Sciences and Guangdong Medical University, was purchased from the Cancer Hospital of Shanghai Fudan University. CNE-2R, a radioresistant human NPC cell line, was previously constructed and maintained at the Cancer Laboratory of Guangxi Medical University. ${ }^{12} \mathrm{CNE}-2 \mathrm{R}$ cells were cultured in RPMI-1640 medium (HyClone, Logan, UT, USA) with 10\% FBS (Thermo Fisher Scientifc, Waltham, MA, USA), penicillin $(100 \mathrm{U} / \mathrm{mL})$, and streptomycin $(100 \mu \mathrm{g} / \mathrm{mL})$ and incubated in a humidified $5 \% \mathrm{CO}_{2}$ atmosphere at $37^{\circ} \mathrm{C}$. The Ethics Committee of the Affiliated Tumor Hospital of Guangxi Medical University approved the study protocol.

\section{Western blotting}

Proteins were extracted 48 hours after the cells were irradiated. The quantitated cell lysates were separated on $8-12 \%$ SDS polyacrylamide gels and electroblotted onto polyvinylidene membranes. After blocking for 1.5 hours, the membranes were incubated sequentially with primary antibodies against LC3B (1:1,000 dilution; \#L7543, Sigma-Aldrich Corp., St. Louis, MO, USA), P62 (1:1,000 dilution; \#A0208, Abcam, Cambridge, UK), and GAPDH antibody (1:1000; \#2118 , Cell Signaling Technology, Boston, MA, USA) at $4{ }^{\circ} \mathrm{C}$ overnight. The membranes were washed and incubated with a secondary antibody (1:15,000 dilution; \#A0208, Beyotime Biotechnology, Shanghai, People's Republic of China) for 1 hour at room temperature. Blotting images of the membranes were obtained using a BioRad Universal Hood III infrared fluorescence imaging system (BioRad Laboratories Inc.,
Hercules, CA, USA). GAPDH served as a control. All experiments were repeated three times.

\section{Cell irradiation and CCK-8 assay}

The Cell Counting Kit-8 (CCK-8) assay was used to assess cell viability after treatment with different doses of X-ray irradiation (IR). Cells were seeded in 96-well plates at $3 \times$ $10^{3}$ cells/well and allowed to attach overnight. Then, they underwent IR with a $6 \mathrm{MV}$ X-ray beam at $2,4,6,8$, or 10 Gy and were cultured for another 48 hours. After treatment, the cells were incubated with $10 \mu \mathrm{g} / \mathrm{mL}$ CCK-8 solution (Dojindo, Japan) for 1 hour in a humidified chamber containing $5 \% \mathrm{CO}_{2}$ at $37^{\circ} \mathrm{C}$. Absorbances were read on a microplate reader (BioRad) at $450 \mathrm{~nm}$. Each group was assessed in five replicate wells, and three independent experiments were conducted. Cell survival was calculated using the following formula: survival rate $(\%)=\mathrm{OD} / \mathrm{OD}_{0 \mathrm{~h}} \times 100 \%$.

\section{Apoptosis assessment}

Cells were irradiated with a $6 \mathrm{MV}$ X-ray beam at a dose of 10 Gy and collected after 48 hours of culture. Then, the samples were stained with $5 \mu \mathrm{L}$ Annexin V PE and $5 \mu \mathrm{L}$ 7-aminoactinomycin D (BD Pharmingen, USA) according to the manufacturer's instructions. Analysis was carried out immediately on an FC500 flow cytometry system. All samples were assessed in triplicate.

\section{Colony-formation assay}

Colony-formation assays were conducted to evaluate the radiosensitivity of cells after IR. Suspensions containing 200, 200, 400,800, 1,000, and 2,000 cells were seeded into five of the six-well plates and individually exposed to doses of $0,2,4,6,8$ and $10 \mathrm{~Gy}$, respectively, with a $6 \mathrm{MV}$ X-ray beam from an Elekta linear accelerator (Precise 1120; Elekta Instrument AB, Stockholm, Sweden) at a dose rate of $220 \mathrm{cGy} / \mathrm{min}$. Then, the cells were incubated for another 14 days until colony appearance. Next, the colonies were fixed with carbinol for 15 minutes and stained with $0.1 \%$ Giemsa (AppliChem, Germany) for 30 minutes. Colonies with more than 50 cells were counted. All experiments were conducted three times. Dose-response curves were analyzed using the multitarget single-hit model in the GraphPad Prism 5.0 software $^{13,14}$ with the formula: Survival fraction $(\mathrm{SF})=1-$ $\left(1-\mathrm{e}^{-D / D 0}\right) N$, where $D_{0}$ (mean lethal dose) is the single dose of radiation that kills $63 \%$ of cells, $N$ represents the number of intracellular radiosensitive areas; $D q\left(\ln \mathrm{N}^{*} \mathrm{D}_{0}\right)$ is the required threshold for cell damage. When the $D q$ increases, the survival curve area is widened, and radioresistance is increased. $S F_{2}$ is an important indicator of cell 
radiosensitivity; the greater the $S F_{2}$, the stronger is the radioresistance.

\section{Statistical analysis}

Data were presented as mean \pm SD. Statistical analyses were conducted using SPSS 17.0 (SPSS, Chicago, IL, USA) or the GraphPad Prism 5.0 software. Statistical differences were determined with a two-tailed $t$-test. ANOVA was used to assess the significance of the differences among the experimental groups. All cell-based experiments were conducted three or four times. A $P$-value $<0.05$ is indicated by *; A $P$-value $<0.01$ is indicated by **.

\section{Results}

\section{Radiosensitivity of the parental CNE-2 and radioresistant CNE-2R cell lines}

Colony-formation assays were conducted to evaluate the radiosensitivity of the CNE-2R and CNE-2 cell lines (Figure 1A). There were more surviving CNE-2R colonies than CNE-2 colonies at doses of 2-10 Gy. Cell survival curves were fitted with the multitarget model in the GraphPad Prism 6.0 software. As shown in Figure 1B, CNE-2R cell survival was higher than CNE-2 survival at 2, 4, 6, 8, and $10 \mathrm{~Gy}$. The main biological parameters associated with radiotherapy are shown in Table 1. Based on the $D q$ and survival fraction at $2 \mathrm{~Gy}\left(\mathrm{SF}_{2}\right)$ values, the repair capability of CNE-2R was stronger than that of CNE-2. Together, these data suggest that CNE-2R is radioresistant compared to CNE-2.

\section{IR induces autophagy in CNE-2R cells}

Western blotting was undertaken to examine the expression levels of autophagy-associated proteins in CNE-2R cells after $0,2,4,6,8$, and 10 Gy IR. As the radiation dose increased, LC3-II protein expression in CNE-2R cells increased, and P62 expression gradually decreased; the levels of LC3-II and P62 became stable after 8-10 Gy IR (Figure 2A). These findings indicate that prompt radiation can activate autophagy in CNE-2R cells and that the level of autophagy reaches a plateau at $8-10$ Gy. Therefore, in subsequent experiments, we used $10 \mathrm{~Gy}$ as the experimental IR dose.

\section{CDP inhibits the level of autophagy induced by IR (IO Gy) in CNE-2R cells, whereas RAPA increases the level of autophagy}

Different concentrations of the autophagy inhibitor CDP $(0,20,40,60$, and $80 \mu \mathrm{mol} / \mathrm{L})$ and the autophagy activator

Table I Correlation parameters in the multitarget single-hit model

\begin{tabular}{|l|l|l|l|}
\hline Cells & $\boldsymbol{D}_{0}(\mathbf{G y})$ & $\boldsymbol{D}_{q}(\mathbf{G y})$ & $\boldsymbol{S F}_{2}$ \\
\hline CNE-2 & $1.123 \pm 0.207$ & $2.187 \pm 0.229$ & $0.418 \pm 0.142$ \\
CNE-2R & $1.647 \pm 0.190$ & $3.73 \pm 0.405$ & $0.714 \pm 0.073$ \\
$P$ & $<0.05$ & $<0.05$ & $<0.05$ \\
\hline
\end{tabular}
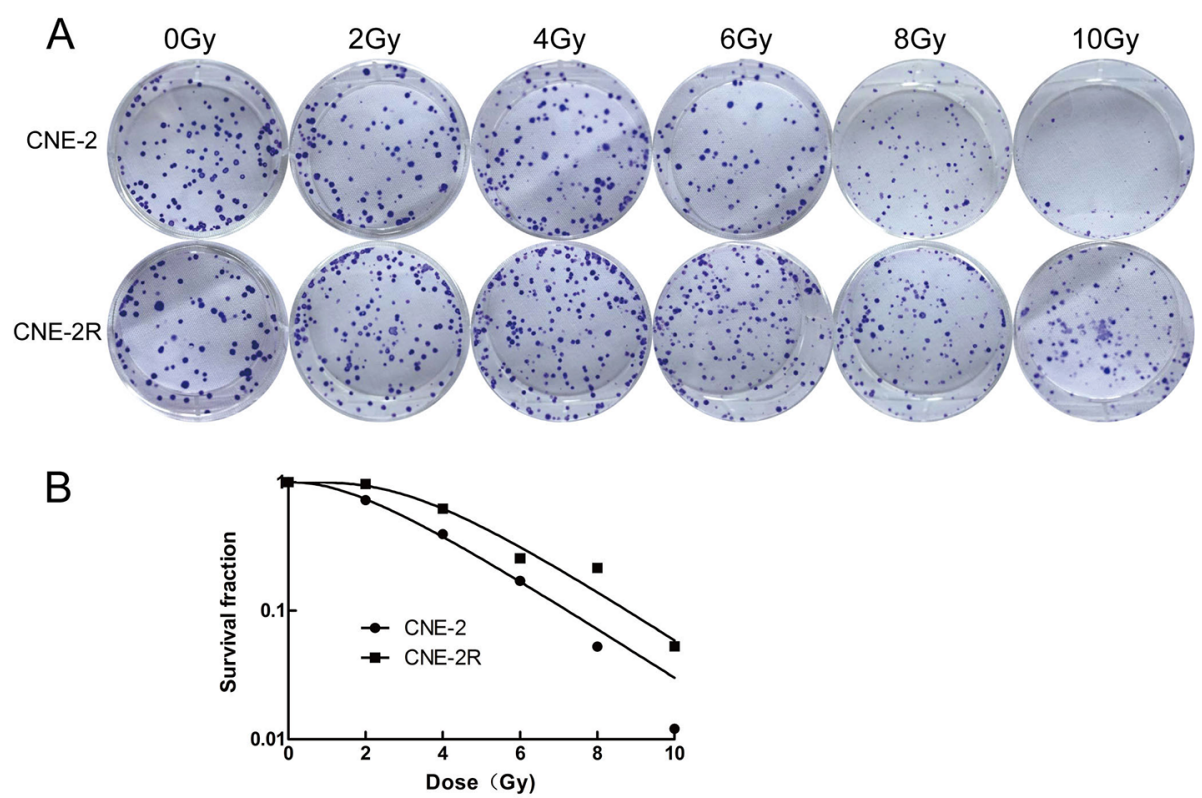

\footnotetext{
Figure I Colony-formation assays assessing the radiosensitivity of the parental CNE-2 and radioresistant CNE-2R cell lines.

Notes: (A) Colony-formation assays of the CNE-2 and CNE-2R cell lines were conducted to evaluate radiosensitivity. (B) The fit curves were generated with the GraphPad Prism 6.0 software. The results were reproduced in three independent experiments.
} 

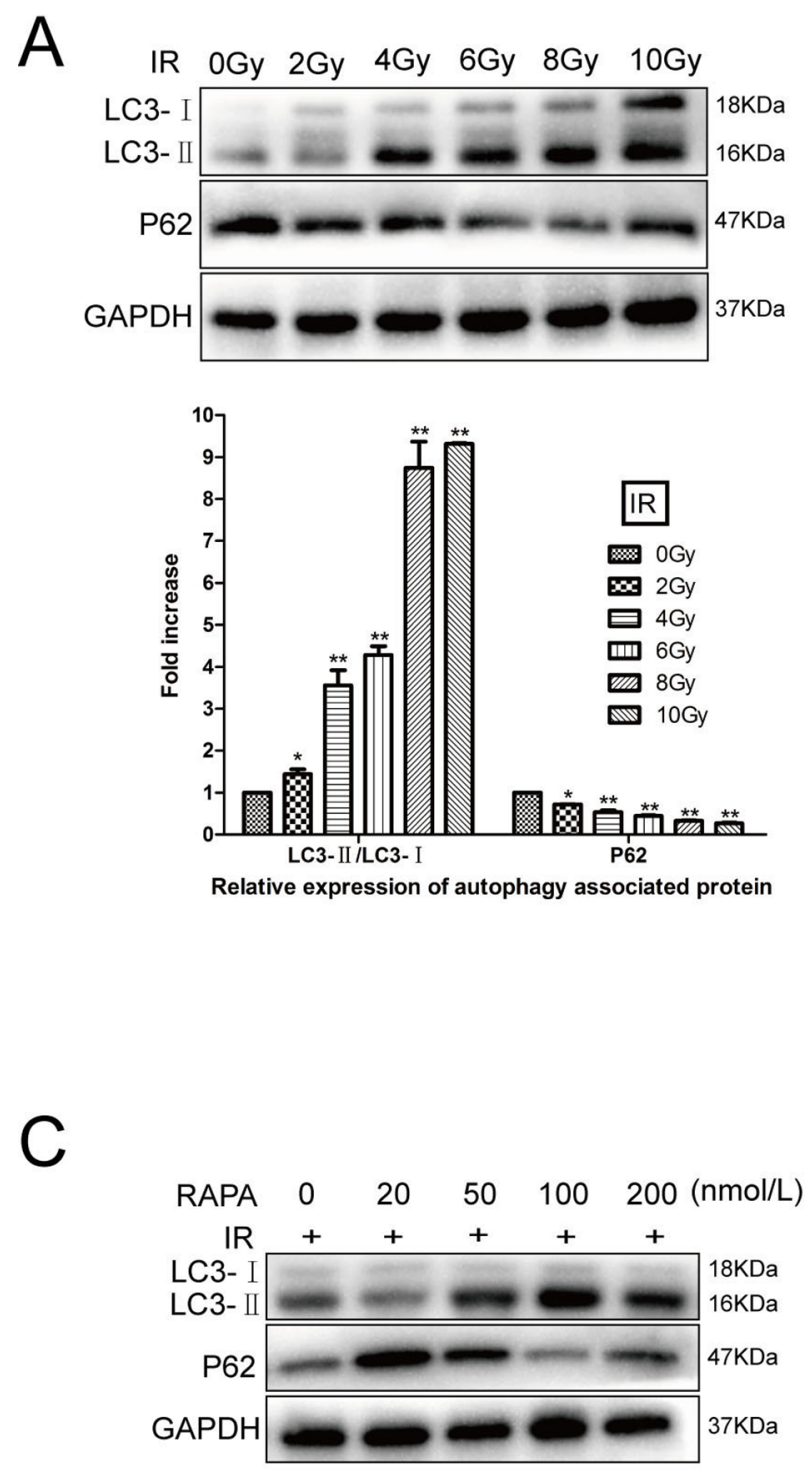

Figure 2 Expression of LC3-I, LC3-II, and P62 in the CNE-2R cells of three groups.

Notes: (A) The CNE-2R cells exposed to different radiation doses. (B) The CNE-2R cells exposed to irradiation (IR; $10 \mathrm{~Gy}$ ) with different concentrations of CDP. (C) The CNE-2R cells exposed to IR ( $10 \mathrm{~Gy}$ ) with different concentrations of rapamycin. All experiments were repeated three times and produced identical findings.

Abbreviations: CDP, chloroquine diphosphate; IR, irradiation; LC3, light chain 3; RAPA, rapamycin.

RAPA $(0,20,50,100$, and $200 \mathrm{nmol} / \mathrm{L})$ were used for CNE2R cells 12 hours before IR ( $10 \mathrm{~Gy}$ ). After 48 hours, proteins were extracted, and Western blotting was undertaken to examine autophagy-related protein expression in CNE-2R cells. Our results showed that autophagy protein expression was inhibited by CDP. At $60-80 \mu \mathrm{mol} / \mathrm{L} \mathrm{CDP}$, the level of LC3-II decreased significantly, and P62 expression increased significantly, indicating that autophagy was clearly inhibited (Figure 2B). With regard to the responses to different concentrations of RAPA, the expression levels of LC3-II and P62 were most notably increased and decreased, respectively,
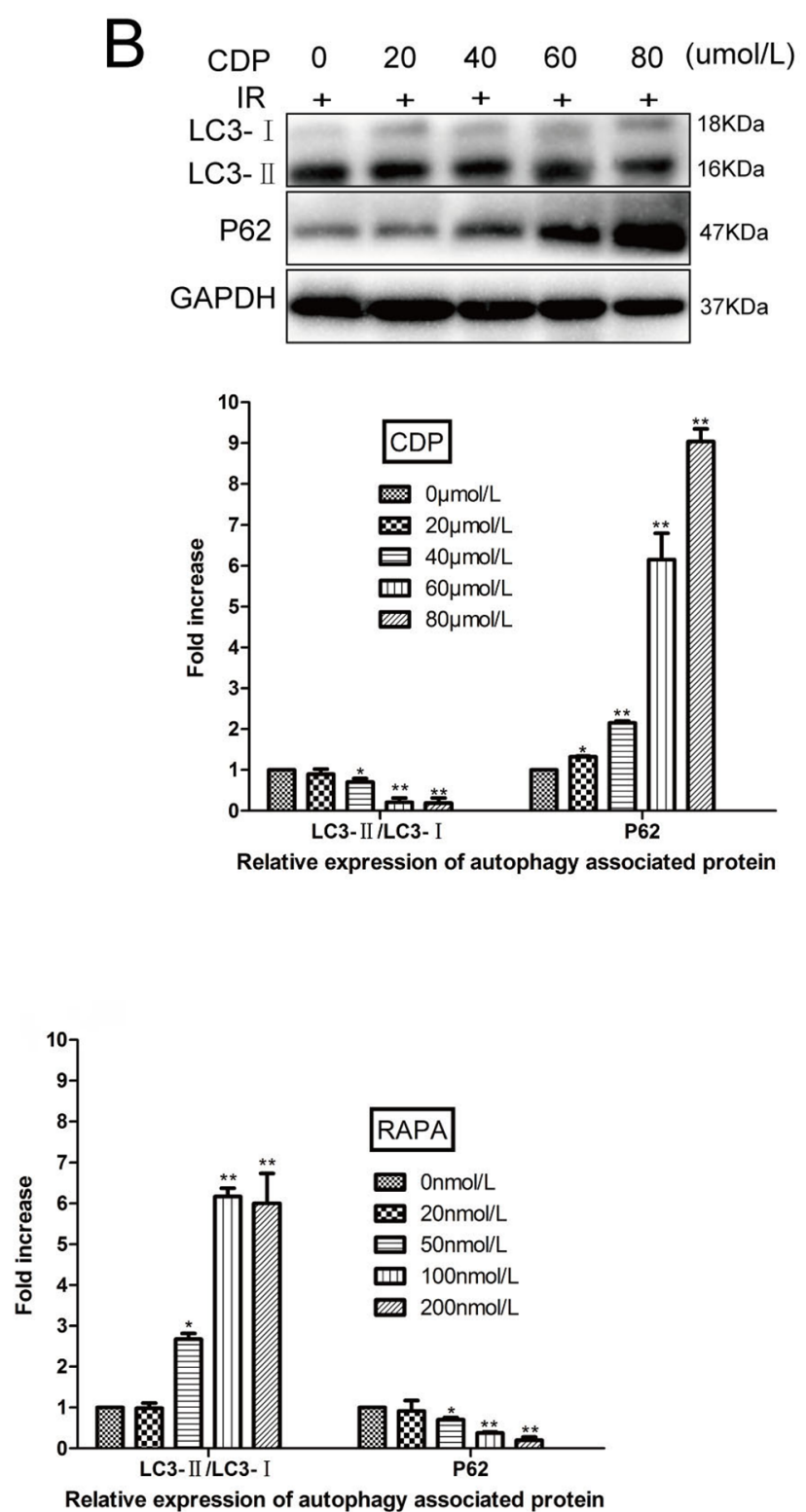

at a concentration of $100 \mathrm{nmol} / \mathrm{L}$, indicating that autophagy was significantly activated (Figure 2C).

\section{Comparison of autophagy levels in response to factors related to different processes}

In the aforementioned portion of the study, we found that 10 Gy radiation, $60 \mu \mathrm{mol} / \mathrm{L} \mathrm{CDP}$, and $100 \mathrm{nmol} / \mathrm{L}$ RAPA had the most significant effects on the autophagy of CNE-2R cells. Therefore, these conditions were used as the experimental conditions. 
In our first analysis, we used untreated CNE-2R cells as the control group. Western blotting experiments were conducted to examine the expression levels of autophagyassociated proteins LC3-II and P62 in the control group, CDP alone group, and RAPA alone group. The levels of LC3-II in the three groups were low, and the levels of P62 protein in all groups were high, with no statistically significant differences ( $P>0.05$; Figure $3 \mathrm{~A})$. This indicated that the autophagy levels of these three groups were extremely low without IR.

In our next analysis, we used untreated CNE-2R cells as the control group. Additionally, the 10-Gy radiotherapy group (the IR alone group), 10-Gy radiotherapy+60- $\mu \mathrm{mol} / \mathrm{L}$ CDP group (the IR+ CDP group), and 10-Gy radiotherapy+100 nmol/L RAPA group (the IR+ RAPA group) were set up. Our results showed that LC3-II expression was significantly suppressed and P62 expression was obviously higher in the IR+ CDP group than in the IR alone group $(P<0.05)$. These results demonstrated that IR-induced autophagy was significantly inhibited by CDP. However, the expression levels of LC3-II were clearly increased, and the expression levels of P62 were significantly decreased in the IR+ RAPA group as compared to the IR alone group, indicating that IR-induced autophagy was clearly enhanced by RAPA (all $P<0.05$; Figure 3 ).

\section{The role of autophagy in the survival of CNE-2R cells}

To assess the role of autophagy in CNE-2R cells, CCK- 8 assays were conducted to assess CNE-2R cell survival after IR. As shown in Figure 4, survival rates were significantly lower for the IR+ CDP group than for the control group at 2, 4, 6, 8, and 10 Gy ( $P<0.05$ for all comparisons) but were significantly higher for the IR+ RAPA group than for the control group at 2, 4, 6, 8, and 10 Gy $(P<0.05$ for all comparisons). The results indicated that autophagy could increase the survival rate of CNE-2R cells.

\section{The effect of autophagy on the apoptosis of CNE-2R cells}

Flow cytometry was used to determine the effects of autophagy on CNE-2R cell apoptosis (Figure 5). The results showed that the incidence of apoptosis was low in the control, CDP alone, and RAPA alone groups without IR. The apoptosis
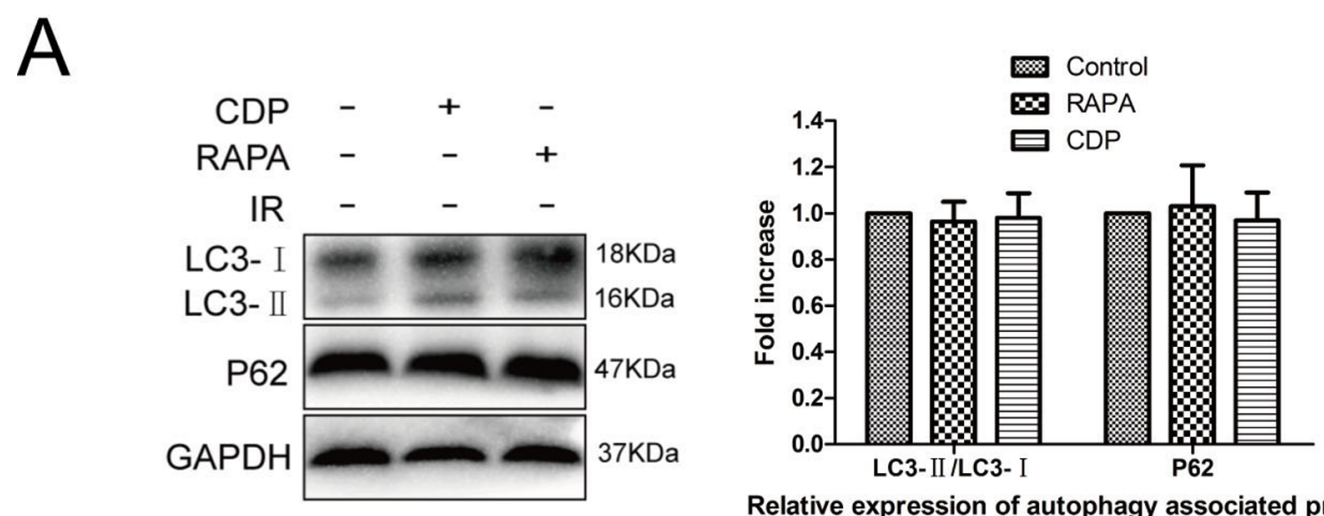

Relative expression of autophagy associated protein
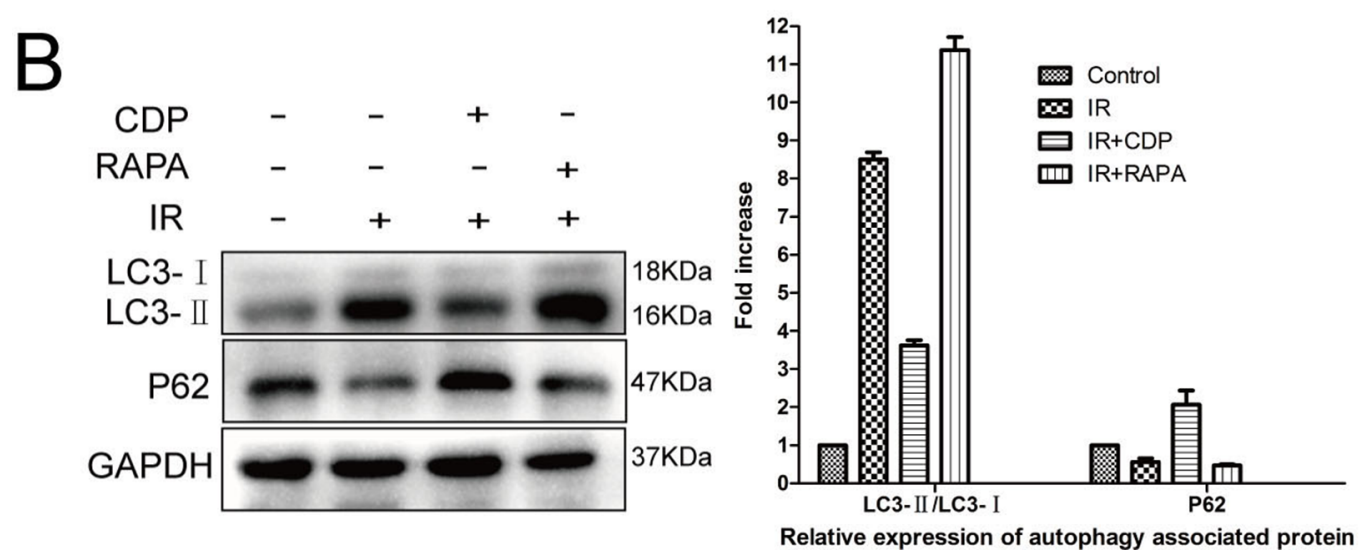

Figure 3 Expression of LC3-I, LC3-II, and P62 in the CNE-2R cells of the different groups.

Notes: (A) The control group, CDP alone group, and rapamycin (RAPA) alone group. (B) The control group, irradiation (IR) alone group, IR+ CDP alone group, and IR+ RAPA group. The results were reproduced in three independent experiments.

Abbreviations: CDP, chloroquine diphosphate; IR, irradiation; LC3, light chain 3; RAPA, rapamycin. 


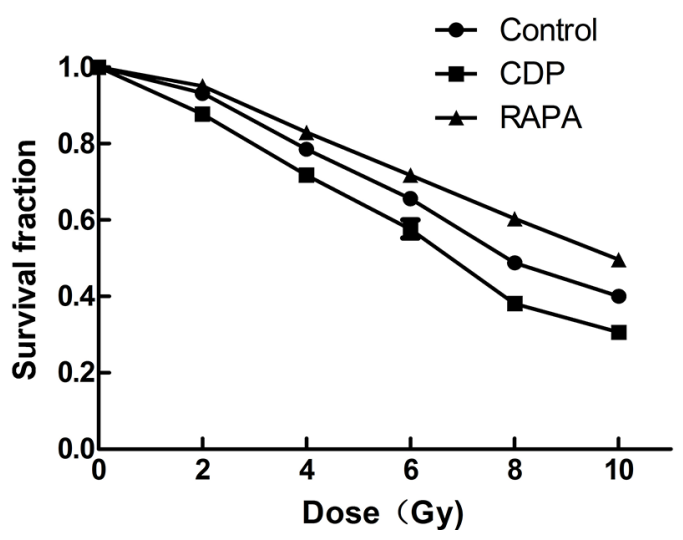

Figure 4 The effects of autophagy on CNE-2R cell survival.

Notes: The CCK- 8 assay was used to assess CNE-2R cell viability. The survival rates of the control, IR + CDP, and IR + RAPA groups were significantly different at the radiation doses of $2,4,6,8$, and 10 Gy (all $P<0.05)$. The results were reproduced in three independent experiments.

Abbreviations: CDP, chloroquine diphosphate; RAPA, rapamycin.

rates for the control, CDP alone, and RAPA alone groups were $3.65 \pm 0.36,4.04 \pm 0.19$, and $3.35 \pm 0.41$, respectively. There were no significant differences in the apoptosis rates of the three groups (all $P>0.05$ ). However, with 10 Gy IR for 48 hours, the apoptosis rates of the control, IR+ CDP, and IR+ RAPA groups were $30.65 \pm 0.68,41.06 \pm 1.02$, and $12.83 \pm 0.68$, respectively (all $P<0.01$ ). These results indicated that the inhibition of autophagy in CNE-2R cells could increase the level of apoptosis, whereas the enhancement of autophagy could decrease the apoptosis rate.

\section{The role of autophagy in the radiosensitivity of CNE-2R cells}

Colony-formation assays were conducted to evaluate the radiosensitivity of CNE-2R cells (Figure 6). Cell survival curves were fitted with the multitarget model in the GraphPad Prism 6.0 software. The survival rate of the IR+ CDP group was lower than that of the control group, whereas the survival rate of the RAPA group was higher than those of the control and CDP groups at 2, 4, 6, 8, and $10 \mathrm{~Gy}$. The main biological parameters associated with radiotherapy are shown in Table 2. Based on the $D q$ and $S F_{2}$ values, we concluded that the higher the autophagy level was, the stronger the survival protection in CNE-2R cells against IR would be. Therefore, autophagy inhibition can increase the sensitivity of CNE-2R cells to IR, whereas autophagy enhancement can reduce radiosensitivity.

\section{Discussion}

The present study showed that the inhibition of autophagy with CDP increased the radiosensitivity of CNE-2R cells, whereas the activation of autophagy with rapamycin (RAPA) decreased the radiosensitivity of CNE-2R cells.

Autophagy is a cellular survival mechanism in which proteins and other cytoplasmic materials are recycled to support cell survival under metabolically stressful conditions, such as hunger, exposure to cytotoxic substances, and exposure to ionizing radiation. LC3 and p62 levels are often used to measure autophagy in cells. ${ }^{15,16} \mathrm{LC} 3$ conversion (LC3-I to LC3-II) and the lysosomal degradation of LC3-II reflect the progression of autophagy. Another widely used autophagy marker, p62, binds directly to LC3 and GABARAP (Atg8 orthologs) family proteins via a short LC3 interaction region (LIR). This may serve as a mechanism to deliver selective autophagic cargo for degradation by autophagy. When autophagy occurs, p62 decreases with protein degradation and intracytoplasmic soluble LC3-I interacts with phosphatidylethanolamine to form LC3-II, leading to a reduction in LC3-I. In the present study, when CNE-2R cells were exposed to IR, the levels of LC3-II/ LC3-I increased, and those of p62 decreased. Therefore, we concluded that autophagy was activated by IR. Moreover, our research showed that the levels of autophagy were boosted by increases in the radiation dose.

Autophagy can help tumor cells eliminate radiation damage, which is an important mechanism of radioresistance. ${ }^{17,18}$ Increasing evidence indicates that the inhibition of autophagy contributes to the sensitivity to anticancer treatments by promoting apoptosis and inhibiting cell proliferation. ${ }^{9} 10$ In 2017, Peng et al found that hepatocellular carcinoma cells with autophagic depletion proliferated much more slowly than control cells after IR exposure. Moreover, after autophagy inhibition, the levels of Bcl-2 remarkably decreased, and the levels of Bax and cleaved caspase- 3 increased. ${ }^{9}$ In 2018 , $\mathrm{Hu}$ et al found that when compared to cells treated with IR alone, treatment with chloroquine, an autophagy inhibitor, sensitized colorectal cancer cells to radiation by increasing apoptosis and reducing the survival rate. ${ }^{10}$ In the present study, we found that cell proliferation was reduced and that cell apoptosis was activated in CNE-2R cells that were exposed to IR when autophagy was inhibited with CDP. In contrast, the activation of autophagy leads to radioresistance during the treatment of cancer. ${ }^{19,20}$ In 2011, Zhuang et al found that RAPA activated autophagy in glioblastoma and that cell proliferation, viability, and clonogenic ability after radiation were reduced. ${ }^{19}$ In 2016, Zhang et al found that the activation of autophagy could decrease the survival rate of non-smallcell lung cancer cells and thus lead to radioresistance. ${ }^{20}$ Similarly, our study showed that the radiosensitivity of CNE-2R 
A

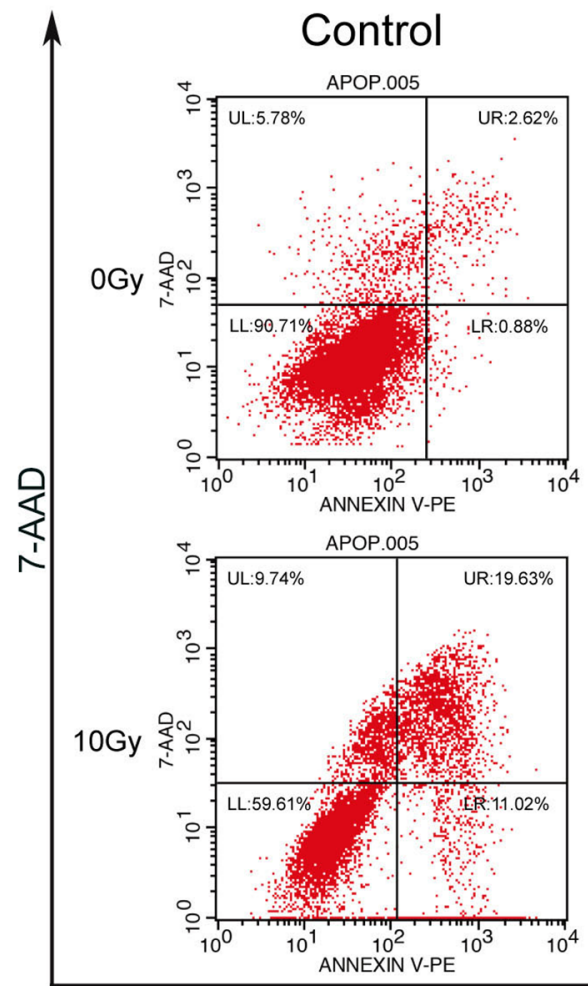

CDP
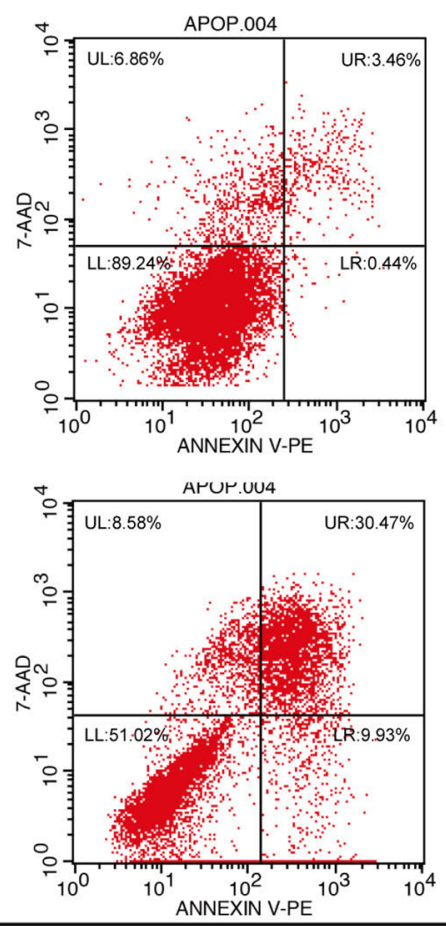

ANNEXIN V-PE
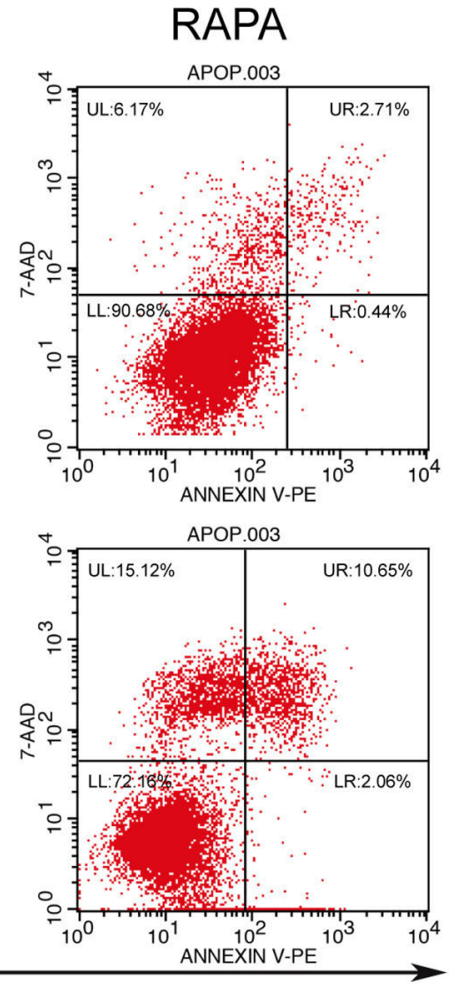

B

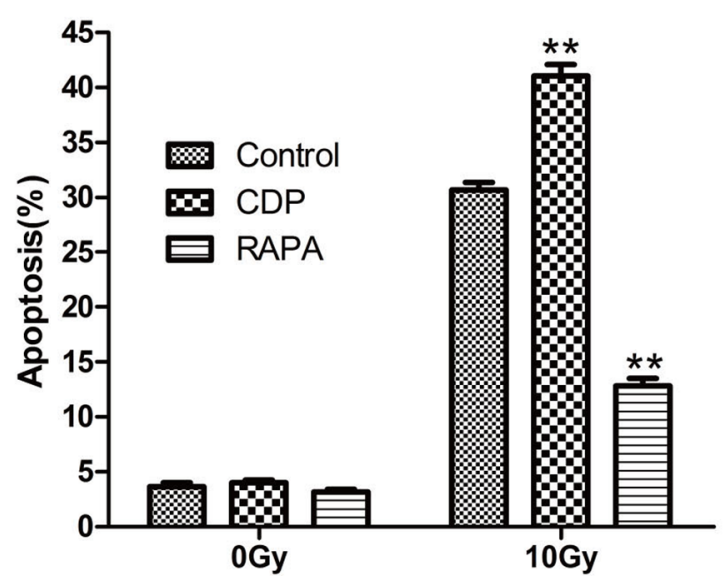

Figure 5 The effects of autophagy on CNE-2R cell apoptosis.

Notes: Apoptotic cells were assessed with Annexin V-PE staining and then subjected to flow cytometry for analysis. (A) Representative images show the Annexin $V-P E$ staining of the control group, CDP alone group, RAPA alone group, IR alone group, IR+CDP alone group, and IR+ RAPA group. (B) Quantification of each group (**P<0.0I). The results were reproduced in three independent experiments.

Abbreviations: CDP, chloroquine diphosphate; RAPA, rapamycin.

cells was decreased by the activation of autophagy, which could enhance proliferation and reduce apoptosis.

Recent studies have shown that chloroquine or RAPA can be applied to change the sensitivity of tumor cells to chemotherapy or radiotherapy via the modulation of autophagy. ${ }^{21,22}$ Due to its chemical properties as a weak base, chloroquine can inhibit H+-ATPase to increase the lysosomal $\mathrm{pH}$, thus inhibiting the enzymatic activity of lysosomal hydrolases and preventing the fusion of the autophagosome and lysosome for lysosomotropic activities. ${ }^{23,24}$ Golden et al suggested that chloroquine blocked autophagy and triggered endoplasmic reticulum stress, thereby increasing the chemosensitivity of glioma cells to temozolomide. ${ }^{25}$ In addition, Ye et al showed that chloroquine enhanced the radiosensitivity of glioma-initiating cells. ${ }^{21}$ The mammalian target of rapamycin (mTOR) is an evolutionarily conserved serine/threonine 

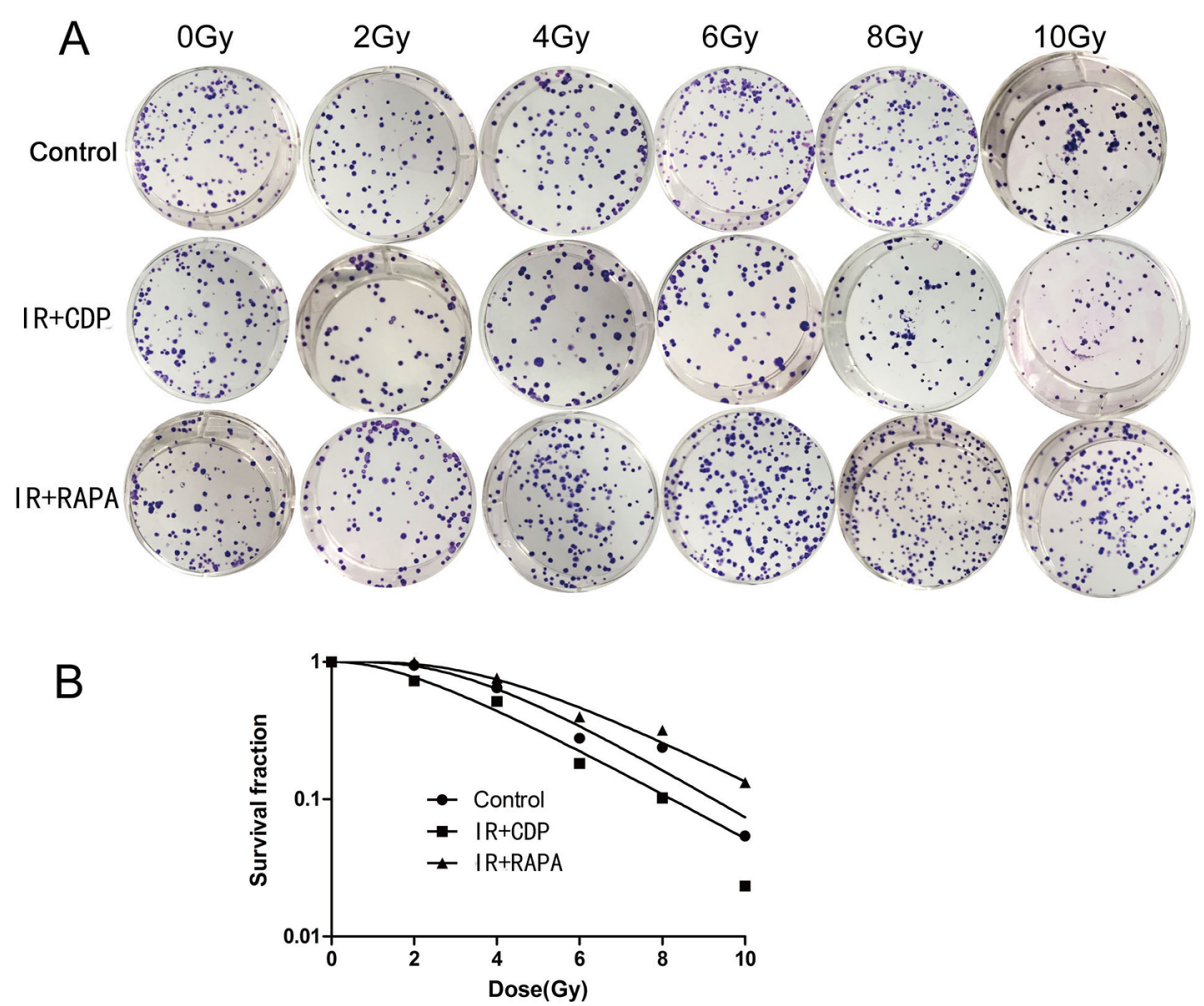

Figure $\mathbf{6}$ The role of autophagy in the radiosensitivity of CNE-2R cells.

Notes: (A) Effect of autophagy on the colony-formation ability of CNE-2R cells under different doses of IR. (B) Fit curves were generated with the GraphPad Prism 6.0 software. The results were reproduced in three independent experiments.

Abbreviations: CDP, chloroquine diphosphate; IR, irradiation; RAPA, rapamycin.

Table 2 Correlation parameters in the multitarget single-hit model

\begin{tabular}{|l|l|l|l|}
\hline Cells & $\boldsymbol{D}_{0}$ & $\mathbf{D q}$ & $\boldsymbol{S F}_{\mathbf{2}}$ \\
\hline Control group & $1.847 \pm 0.125$ & $3.725 \pm 0.30 \mathrm{I}$ & $0.650 \pm 0.143$ \\
IR+ CDP group & $1.352 \pm 0.27 \mathrm{I}$ & $2.102 \pm 0.45 \mathrm{I}$ & $0.46 \mathrm{I} \pm 0.203$ \\
IR+ RAPA group & $2.415 \pm 0.285$ & $5.422 \pm 0.318$ & $0.836 \pm 0.062$ \\
$P$ & $<0.05$ & $<0.05$ & $<0.05$ \\
\hline
\end{tabular}

Abbreviations: IR, irradiation; CDP, chloroquine diphosphate; RAPA, rapamycin.

kinase and the founding member of a signaling pathway that regulates many fundamental features of cell growth and division. ${ }^{26}$ RAPA, a specific inhibitor of mTOR, can activate autophagy by inhibiting the mTOR pathway and plays a role in tumor inhibition. ${ }^{27} \mathrm{Li}$ et al found that RAPA-induced autophagy sensitizes lung cancer cells to radiation associated with DNA damage repair inhibition. ${ }^{28}$ Moreover, the present study proved that both chloroquine and RAPA changed the radiosensitivity of CNE-2R cells by regulating autophagy. Moreover, we found that the autophagy of CNE-2R cells exposed to IR was significantly inhibited by $60 \mu \mathrm{mol} / \mathrm{L} \mathrm{CDP}$ and was notably activated by $100 \mathrm{nmol} / \mathrm{L}$ RAPA. In a study by Wang et al, a low concentration of chloroquine $(10 \mu \mathrm{M})$ was applied to investigate its influence on the radiosensitivity of bladder cancer cells. ${ }^{29}$ However, the study by Ye et al found that a specific dose of chloroquine $(20 \mathrm{nmol} / \mathrm{L})$ clearly enhanced the radiosensitivity of glioma-initiating cells by inhibiting autophagy. ${ }^{21}$ Dai et al confirmed that autophagy was significantly induced by RAPA $(50 \mathrm{nmol} / \mathrm{L})$ in pancreatic cancer cells. ${ }^{30}$ Therefore, we speculate that, for the different kinds of cancer cells, CDP and RAPA might have different optimal drug concentrations to inhibit or activate autophagy.

\section{Conclusion}

We have demonstrated that the level of autophagy has a negative correlation with the radiosensitivity of the radioresistant human NPC cell line CNE-2R. In addition, either chloroquine or RAPA can be utilized to change the sensitivity of CNE-2R to radiotherapy via the modulation of autophagy. Further animal experiments and clinical tests are warranted to verify our findings.

\section{Acknowledgments}

This work was sponsored by grants from the National Natural Science Foundation of China (grant nos. 81160285 and 81760544), the Basic Ability Enhancement Project of Young Teachers in Guangxi Zhuang Autonomous Region (grant no. 2017KY0114), the Youth Science Foundation of Guangxi Medical University (grant no. GXMUYSF201515), and the 
International Communication of Guangxi Medical University Graduate Education in 2018.

\section{Author contributions}

Xiao-Dong Zhu and Zhong-Guo Liang conceived and designed the experiments; Zhong-Guo Liang, Guo-Xiang Lin, and Bin-Bin Yu conducted the experiments; Fang Su, Ling Li, and Song Qu analyzed the data; and Zhong-Guo Liang and Guo-Xiang Lin wrote the paper. Zhong-Guo Liang, Guo-Xiang Lin, and Bin-Bin Yu contributed equally to this study and are co-first authors of this manuscript. All authors contributed to data analysis, drafting and revising the article, gave final approval of the version to be published, and agree to be accountable for all aspects of the work.

\section{Disclosure}

The authors report no conflicts of interest in this work.

\section{References}

1. Torre LA, Bray F, Siegel RL, Ferlay J, Lortet-Tieulent J, Jemal A. Global cancer statistics, 2012. CA Cancer J Clin. 2015;65(2):87-108.

2. Tang LL, Chen WQ, Xue WQ, et al. Global trends in incidence and mortality of nasopharyngeal carcinoma. Cancer Lett. 2016;374(1):22-30.

3. Au KH, Ngan RKC, Ng AWY, et al. Treatment outcomes of nasopharyngeal carcinoma in modern era after intensity modulated radiotherapy (IMRT) in Hong Kong: A report of 3328 patients (HKNPCSG 1301 study. Oral Oncol. 2018;77:16-21.

4. Lee AW, Ma BB, Ng WT, Chan AT. Management of Nasopharyngeal Carcinoma: Current Practice and Future Perspective. J Clin Oncol. 2015;33(29):3356-3364.

5. Kong F, Ying H, du C, et al. Patterns of local-regional failure after primary intensity modulated radiotherapy for nasopharyngeal carcinoma. Radiat Oncol. 2014;9:60.

6. Li YQ, Tian YM, Tan SH, et al. Prognostic Model for Stratification of Radioresistant Nasopharynx Carcinoma to Curative Salvage Radiotherapy. J Clin Oncol. 2018;36(9):891-899.

7. Hönscheid P, Datta K, Muders MH. Autophagy: detection, regulation and its role in cancer and therapy response. Int J Radiat Biol. 2014;90(8):628-635.

8. Xu Z, Yan Y, Zeng S, et al. Reducing autophagy and inducing G1 phase arrest by aloperine enhances radio-sensitivity in lung cancer cells. Oncol Rep. 2017.

9. Peng WX, Wan YY, Gong AH, et al. Egr-1 regulates irradiation-induced autophagy through Atg $4 \mathrm{~B}$ to promote radioresistance in hepatocellular carcinoma cells. Oncogenesis. 2017;6(1):e292.

10. Hu JL, He GY, Lan XL, et al. Inhibition of ATG12-mediated autophagy by miR-214 enhances radiosensitivity in colorectal cancer. Oncogenesis. 2018;7(2):16.

11. Zhou ZR, Zhu XD, Zhao W, et al. Poly(ADP-ribose) polymerase-1 regulates the mechanism of irradiation-induced CNE-2 human nasopharyngeal carcinoma cell autophagy and inhibition of autophagy contributes to the radiation sensitization of CNE-2 cells. Oncol Rep. 2013;29(6):2498-2506.
12. Guo Y, Zhu XD, Qu S, et al. Identification of genes involved in radioresistance of nasopharyngeal carcinoma by integrating gene ontology and protein-protein interaction networks. Int J Oncol. 2012;40(1):85-92.

13. Guo SI-YAN, Zhu X-D, Ge L-Y, et al. RNAi-mediated knockdown of the c-jun gene sensitizes radioresistant human nasopharyngeal carcinoma cell line CNE-2R to radiation. Oncol Rep. 2015;33(3):1155-1160.

14. Lin H, Chen ZT, Zhu XD, et al. Serum CD166: A novel biomarker for predicting nasopharyngeal carcinoma response to radiotherapy. Oncotarget. 2017;8(38):62858-62867.

15. Jiang P, Mizushima N. LC3- and p62-based biochemical methods for the analysis of autophagy progression in mammalian cells. Methods. 2015;75:13-18.

16. Schläfli AM, Berezowska S, Adams O, Langer R, Tschan MP. Reliable LC3 and p62 autophagy marker detection in formalin fixed paraffin embedded human tissue by immunohistochemistry. Eur J Histochem. 2015;59(2):2481.

17. Xin Y, Jiang F, Yang C, et al. Role of autophagy in regulating the radiosensitivity of tumor cells. J Cancer Res Clin Oncol. 2017;143(11):2147-2157.

18. das CK, Mandal M, Kögel D. Pro-survival autophagy and cancer cell resistance to therapy. Cancer Metastasis Rev. 2018.

19. Zhuang W, Li B, Long L, Chen L, Huang Q, Liang Z. Induction of autophagy promotes differentiation of glioma-initiating cells and their radiosensitivity. Int J Cancer. 2011;129(11):2720-2731.

20. Zhang X, Ji J, Yang Y, Zhang J, Shen L. Stathmin1 increases radioresistance by enhancing autophagy in non-small-cell lung cancer cells. Onco Targets Ther. 2016;9:2565-2574.

21. Ye H, Chen M, Cao F, Huang H, Zhan R, Zheng X. Chloroquine, an autophagy inhibitor, potentiates the radiosensitivity of glioma initiating cells by inhibiting autophagy and activating apoptosis. BMC Neurol. 2016;16(1):178.

22. Kaur A, Sharma S. Mammalian target of rapamycin (mTOR) as a potential therapeutic target in various diseases. Inflammopharmacology. 2017;25(3):293-312.

23. Rubinsztein DC, Codogno P, Levine B. Autophagy modulation as a potential therapeutic target for diverse diseases. Nat Rev Drug Discov. 2012;11(9):709-730.

24. Avniel-Polak S, Leibowitz G, Riahi Y, Glaser B, Gross DJ, GrozinskyGlasberg S. Abrogation of Autophagy by Chloroquine Alone or in Combination with mTOR Inhibitors Induces Apoptosis in Neuroendocrine Tumor Cells. Neuroendocrinology. 2016;103(6):724-737.

25. Golden EB, Cho HY, Jahanian A, et al. Chloroquine enhances temozolomide cytotoxicity in malignant gliomas by blocking autophagy. Neurosurg Focus. 2014;37(6):E12.

26. Liu Q, Thoreen C, Wang J, Sabatini D, Gray NS. mTOR Mediated Anti-Cancer Drug Discovery. Drug Discov Today Ther Strateg. 2009;6(2):47-55.

27. Weichhart T. Mammalian target of rapamycin: a signaling kinase for every aspect of cellular life. Methods Mol Biol. 2012;821:1-14.

28. Li Y, Liu F, Wang Y, et al. Rapamycin-induced autophagy sensitizes A549 cells to radiation associated with DNA damage repair inhibition. Thorac Cancer. 2016;7(4):379-386.

29. Wang F, Tang J, Li P, et al. Chloroquine Enhances the Radiosensitivity of Bladder Cancer Cells by Inhibiting Autophagy and Activating Apoptosis. Cell Physiol Biochem. 2018;45(1):54-66.

30. Dai Z-J, Gao J, Ma X-B, et al. Antitumor effects of rapamycin in pancreatic cancer cells by inducing apoptosis and autophagy. Int J Mol Sci. 2012;14(1):273-285. 


\section{Publish your work in this journal}

Cancer Management and Research is an international, peer-reviewed open access journal focusing on cancer research and the optimal use of preventative and integrated treatment interventions to achieve improved outcomes, enhanced survival and quality of life for the cancer patient. The manuscript management system is completely online and includes a very quick and fair peer-review system, which is all easy to use. Visit http://www.dovepress.com/testimonials.php to read real quotes from published authors.

Submit your manuscript here: https://www.dovepress.com/cancer-management-and-research-journal 